Arq. Bras. Med. Vet. Zootec., v.68, n.3, p.785-794, 2016

\title{
Capacidade produtiva e qualidade nutricional de gramíneas perenes submetidas a sistema contínuo de cortes
}

\author{
[Productive capacity and nutritional quality of different perennial grasses \\ submitted to a continued cuts system] \\ M. Poczynek ${ }^{1}$, M. Neumann ${ }^{1}$, E.H. Horst ${ }^{1}$, G.F.M. Leão ${ }^{2}$, M. Poczynek ${ }^{1}$, R.K. Ueno ${ }^{3}$ \\ ${ }^{1}$ Universidade Estadual do Centro-Oeste - Unicentro - Guarapuava, PR \\ ${ }^{2}$ Universidade Estadual de Maringá- UEM - Maringá, PR \\ ${ }^{3}$ Universidade Federal do Rio Grande do Sul- UFRGS - Porto Alegre, RS
}

\begin{abstract}
RESUMO
O objetivo do trabalho foi avaliar a produtividade e as características bromatológicas dos estratos superior e inferior de gramíneas perenes em sistema contínuo de cortes. As cultivares avaliadas foram: Cynodon nlemfuensis Vanderyst cv. Estrela-roxa; Cynodon dactilon (L.) Pears cv. Coast-cross 1; Cynodon nlemfuensis Vanderyst cv. Tifton 68; Cynodon ssp. cv. Tifton 85; Cynodon dactilon sp. cv. Jiggs; Hemárthria altissima cv. Roxinha e Penissetum clandestinum cv. Quicuio. Todos os cortes foram realizados no momento em que cada cultivar alcançava $95 \%$ de interceptação luminosa. As cutivares Jiggs e Tifton 68, com 21.348 e $21.016 \mathrm{~kg} \mathrm{ha}^{-1}$, respectivamente, tiveram as maiores produções $(\mathrm{P}<0,05)$ de fitomassa seca acumulada. Na média geral dos cortes, o quarto corte apresentou a menor relação estrato superior:inferior em relação aos demais $(\mathrm{P}<0,05)$. Em relação ao estrato superior, a Tifton 85 apresentou o maior teor de $\mathrm{PB}$, sendo superior estatisticamente $(\mathrm{P}<0,05)$ no primeiro, segundo e quarto cortes, enquanto no terceiro corte a Coast-cross apresentou o maior teor proteico. No geral, as cv. Jiggs e Tifton 68 apresentaram a maior produção de fitomassa acumulada. Já a cv. Tifton 85 foi responsável pelos maiores níveis de $\mathrm{PB}$, enquanto os teores de carboidratos estruturais mantiveram padrão constante, tanto no estrato superior quanto inferior, em sistema de quatro cortes sucessivos.
\end{abstract}

Palavras-chave: bromatologia, Cynodon, forragem, pastagem

\begin{abstract}
The aim of this study was to evaluate the productivity and the bromatologic characteristics of the upper and lower strata of perennial grasses in four cut systems. The cultivars evaluated were: Cynodon nlemfuensis Vanderyst cv. Estrela Roxa; Cynodon dactilon (L.) Pears cv. Coast-cross 1; Cynodon nlemfuensis Vanderyst cv. Tifton-68; Cynodon ssp. cv. Tifton-85; Cynodon dactilon sp. cv. Jiggs; Hemárthria altissima cv. Roxinha, and Penissetum clandestinum cv. Quicuio. All cuts were made when each cv. reached 95\% light interception. The cv. Jiggs and Tifton 68, with 21348 and 21016kg ha-1, respectively, were responsible for the higher yields $(P<0.05)$ of accumulated dry biomass. The overall average of the cuts, the fourth cut had the lowest ratio upper stratum: lower compared to the others $(P<0.05)$. Regarding the upper stratum, the $\mathrm{cv}$. Tifton 85 had the highest $C P$ content, statistically higher $(P<0.05)$ in the first, second and fourth cut, while in the third cut the cv. Coast-cross had the highest protein content. In general, $\mathrm{cv}$. Jiggs and Tifton 68 had the highest number of accumulated biomass. The cv. Tifton 85 was responsible for the higher levels of CP, whereas the levels of structural carbohydrates maintained a constant pattern, in both the upper and lower strata in four cut systems.
\end{abstract}

Keywords:bromatology, Cynodon, forage, grass

Recebido em 24 de setembro de 2015

Aceito em 8 de janeiro de 2016

E-mail: mpoczynek@hotmail.com 


\section{INTRODUÇÃO}

Várias espécies e cultivares forrageiras são disponibilizadas por empresas e instituições para atender a demanda dos pecuaristas, porém existe uma grande carência de elucidação de dúvidas inerentes às características das pastagens existentes, como, por exemplo, as espécies forrageiras mais produtivas, bem como as características nutricionais e morfofisiológicas de cada planta, questões essas fundamentais para se fazer um planejamento forrageiro.

Alguns planejamentos forrageiros se valem de sistemas que trabalham com a entrada de um grupo de animais mais exigente nutricionalmente para consumir o estrato superior das plantas e, em seguida, um grupo de animais menos exigente para consumir o estrato inferior delas (Carvalho et al., 2010), visando à otimização da produtividade.

É de conhecimento que as características morfofisiológicas de uma planta influenciam a distribuição e a composição de substratos ao longo do seu perfil estrutural, o que pode afetar a digestibilidade e o consumo diário dos animais conforme o estrato pastejado. Naturalmente, animais pastejam preferencialmente o estrato superior das forragens, dada sua maior concentração de nutrientes, porém, à medida que o dossel é rebaixado, tem-se maior concentração de colmo, sendo essa uma porção mais lignificada (Hodgson, 1981). Assim, os animais passam a ser mais seletivos, e a produção animal tende a declinar.

Segundo Van Soest (1994), o valor nutricional de forragens de clima tropical varia muito de acordo com a maturidade, a relação folha:colmo, além da bromatologia das folhas e colmos. Já o intervalo entre cortes contribui não só para determinar a estabilidade qualitativa da forragem, mas também sua produtividade.

Ademais, estudos ligados à morfologia e aos estratos de plantas têm sido bastante desenvolvidos em espécies forrageiras temperadas, entretanto a literatura é escassa quanto às espécies forrageiras tropicais. Assim, o objetivo deste trabalho foi avaliar a produtividade por cortes e acumulada, a relação entre estrato superior $\mathrm{e}$ inferior quanto à qualidade nutricional de diferentes forrageiras estivais em sistema contínuo de cortes.

\section{MATERIAL E MÉTODOS}

O experimento foi realizado na Universidade Estadual do Centro-Oeste (Unicentro), localizada no município de Guarapuava - PR. O clima da região, segundo a classificação de Kottek, é o $\mathrm{Cfb}$ (subtropical mesotérmico úmido), com verões amenos e invernos moderados, sem estação seca definida e com geadas severas.

O experimento foi conduzido em delineamento de blocos ao acaso, composto por 28 tratamentos em esquema fatorial $7 \times 4$, com 4 repetições. Foram utilizadas cinco forrageiras estivais do gênero Cynodon, sendo: Cynodon nlemfuensis Vanderyst cv. Estrela-roxa; Cynodon dactilon (L.) Pears cv. Coast-cross 1; Cynodon nlemfuensis Vanderyst cv. Tifton 68; Cynodon ssp. cv. Tifton 85 e Cynodon dactilon sp. cv. Jiggs, uma do gênero Hemárthria (Hemárthria altissima cv. Roxinha) e uma do gênero Penissetum (Penissetum clandestinum cv. Quicuio), em um sistema de quatro cortes sucessivos.

O solo da área experimental foi classificado como Latossolo Bruno Típico (POTT, 2007) e, antes da implantação da cultura, apresentava as seguintes características químicas (perfil de 0 a 20cm): $\mathrm{pH} \mathrm{CaCl}{ }_{2} 0,01 \mathrm{M}: 4,6 ; \mathrm{P}: 2,0 \mathrm{mg} \mathrm{dm}^{-3} ; \mathrm{K}^{+}$: 0,43 $\mathrm{cmol}_{\mathrm{c}} \mathrm{dm}^{-3}$; $\mathrm{MO}: 34,9 \mathrm{~g} \mathrm{dm}^{-3} ; \mathrm{Al}^{3+}: 0,0 \mathrm{cmol}_{\mathrm{c}}$ $\mathrm{dm}^{3} ; \mathrm{H}^{+}+\mathrm{Al}^{3+}: 6,4 \mathrm{cmol}_{\mathrm{c}} \mathrm{dm}^{-3} ; \mathrm{Ca}^{2+}: 2,5 \mathrm{cmol}_{\mathrm{c}}$ $\mathrm{dm}^{-3} ; \mathrm{Mg}^{2+}: 2,8 \mathrm{cmol}_{\mathrm{c}} \mathrm{dm}^{-3}$ e saturação de bases (V\%): 48,9\%.

Em um período de 60 dias antes do plantio das mudas, a área foi corrigida para saturação por bases de $70 \%$, com aplicação de calcário calcítico na dose de $2,5 \mathrm{t} \mathrm{ha}^{-1}$. Já na ocasião do plantio, foi realizada uma aplicação de $300 \mathrm{~kg} \mathrm{ha}^{-}$ do fertilizante formulado 04-20-20 (N-P $\mathrm{O}_{5^{-}}$ $\mathrm{K}_{2} \mathrm{O}$ ) conforme recomendação (CQFS RS/SC, 2004). A adubação de cobertura constou de $300 \mathrm{~kg}$ de $\mathrm{N} \mathrm{ha}^{-1}$, realizada em quatro aplicações, sendo a primeira após o corte de uniformização e as outras após cada corte de avaliação.

A implantação das mudas foi realizada em sulcos, com espaçamento entre linhas de $0,25 \mathrm{~m}$, com as mudas dispostas uma das outras a uma distância de $0,25 \mathrm{~m}$. A área de cada parcela foi de $4 \mathrm{~m}^{2}(2,0 \times 2,0 \mathrm{~m})$, sendo considerada como área útil para a coleta de dados a parte central de $2,25 \mathrm{~m}^{2}(1,5 \times 1,5 \mathrm{~m})$, em que cada parcela correspondeu a um tratamento. 
Foi efetuado um corte de uniformização em todas as cultivares no dia $01 / 11 / 2013$. A altura do dossel foi mensurada imediatamente antes e depois de cada corte, seguindo metodologia proposta por Bircham (1981). A Tab. 1 apresenta o intervalo entre os cortes e o ciclo, em dias, de cada cultivar avaliada.

Tabela 1. Intervalo entre cortes e ciclo, em dias, das cultivares perenes avaliadas

\begin{tabular}{lccccc}
\hline \multirow{2}{*}{ Cultivar } & \multicolumn{4}{c}{ Intervalo entre corte, dias } & \multirow{2}{*}{ Ciclo, dias } \\
\cline { 2 - 5 } & $1^{\mathbf{0}}$ corte & $2^{\circ}$ corte & $3^{\circ}$ corte & $4^{\circ}$ corte & \\
\hline Estrela-roxa & 34 & 39 & 49 & 70 & 192 \\
Coast-cross & 33 & 40 & 47 & 66 & 186 \\
Tifton 68 & 33 & 37 & 50 & 46 & 166 \\
Tifton 85 & 35 & 35 & 31 & 35 & 136 \\
Jiggs & 35 & 38 & 47 & 44 & 164 \\
Hemárthria roxinha & 33 & 44 & 59 & 68 & 204 \\
Quicuio & 55 & 36 & 45 & 68 & 204 \\
\hline
\end{tabular}

Tanto a mensuração do índice de área foliar (IAF) do dossel quanto o corte de cada cultivar para obtenção da produção de fitomassa $\mathrm{ha}^{-1}$ foram realizados no momento em que elas alcançaram 95\% de interceptação luminosa (IL), sendo essa estimada por meio da radiação fotossinteticamente ativa (RAF), medida pelo ceptômetro linear digital modelo AccuPAR LP80, (Decagon, Devices). O corte de cada cultivar foi realizado de forma manual, com auxílio de uma foice curva, a uma altura de $0,12 \mathrm{~m}$ do solo.

Uma subamostra de homogênea de cada parcela foi coletada e levada imediatamente ao laboratório para fragmentação em estrato superior (porção superior a $0,12 \mathrm{~m}$ ) e inferior (porção de 0 a $0,12 \mathrm{~m}$ ). Posteriormente, todas as amostras foram pré-secas em estufa de ar forçado a $55^{\circ} \mathrm{C}$ até atingirem peso constante (AOAC, 1995), o que permitiu estimar a produção de fitomassa seca ha ${ }^{-1}$ de cada estrato. Já a relação folha:colmo foi obtida pela razão entre a mensuração da quantidade de colmo e de lâmina foliar avaliados nas diferentes espécies ao longo dos cortes.

As amostras pré-secas foram moídas em moinho tipo "Wiley", com peneira de $1 \mathrm{~mm}$, e, em seguida, procedeu-se à determinação de proteína bruta (PB) pelo método micro- Kjedahl, dos teores da fibra insolúvel em detergente neutro (FDN) conforme Van Soest et al. (1991) e da fibra insolúvel em detergente ácido (FDA) segundo Goering e Van Soest (1970).

Os dados foram submetidos aos testes de Shapiro-Wilk e Bartlett, a fim de se verificarem os pressupostos de normalidade e homogeneidade de variância, respectivamente. Uma vez atendidos esses pressupostos, aplicouse o teste $\mathrm{F}$ a $5 \%$ de probabilidade de confiança, por meio da análise de variância (ANOVA) e, em seguida, o teste de Tukey de comparação de múltiplas médias a $5 \%$ de significância, por intermédio do programa SAS (1993).

\section{RESULTADOS E DISCUSSÃO}

Conforme os dados apresentados na Tab.2, observa-se que a cv. Tifton 68 apresentou a maior produtividade, sendo superior $(\mathrm{P}<0,05)$ a todas as demais no primeiro corte, porém não diferindo $(\mathrm{P}>0,05)$ da cv. Jiggs nos demais cortes. Ao se observar a média geral dos cortes, nota-se comportamento indefinido, tendo o primeiro e o terceiro corte superioridade $(\mathrm{P}<0,05)$ produtiva em relação ao segundo e ao quarto corte, onde as duas cv. supracitadas se destacaram pelo comportamento constante.

De acordo com Pedreira (2009), os estolões vultuosos da cv. Tifton 68 possuem alta capacidade de estocagem de carboidratos solúveis, o que lhe permite uma capacidade rápida de rebrota e maior resistência ao frio, sendo por isso indicada por Burton (1993) a climas subtropicais. Igualmente, a cv. Jiggs, apesar de pouco estudada em solos brasileiros, demonstra potencial produtivo promissor quando comparada a cultivares mais consagradas, corroborando a tese defendida por Guimarães (2012). 
Tabela 2. Produção de fitomassa seca das cultivares perenes, conforme número de cortes

\begin{tabular}{lccccc}
\hline Forrageira & \multicolumn{5}{c}{ Produção de fitomassa seca por corte, kg ha ${ }^{-1}$} \\
\cline { 2 - 6 } & $1^{\circ}$ corte & $2^{\circ}$ corte & $3^{\circ}$ corte & $4^{\circ}$ corte & Acumulado \\
\hline Estrela-roxa & $4.268 \mathrm{de}$ & $3.394 \mathrm{~b}$ & $5.109 \mathrm{~b}$ & $4.556 \mathrm{a}$ & $17.327 \mathrm{bc}$ \\
Coast-cross & $4.837 \mathrm{bcd}$ & $3.687 \mathrm{ab}$ & $3.941 \mathrm{c}$ & $3.347 \mathrm{bc}$ & $15.812 \mathrm{bc}$ \\
Tifton 68 & $6.777 \mathrm{a}$ & $3.646 \mathrm{ab}$ & $6.254 \mathrm{a}$ & $4.339 \mathrm{ab}$ & $21.016 \mathrm{a}$ \\
Tifton 85 & $5.348 \mathrm{bc}$ & $3.935 \mathrm{ab}$ & $4.830 \mathrm{bc}$ & $3.305 \mathrm{c}$ & $17.418 \mathrm{~b}$ \\
Jiggs & $5.749 \mathrm{~b}$ & $4.606 \mathrm{a}$ & $6.333 \mathrm{a}$ & $4.661 \mathrm{a}$ & $21.348 \mathrm{a}$ \\
Hemártrhia roxinha & $3.646 \mathrm{e}$ & $3.130 \mathrm{~b}$ & $4.587 \mathrm{bc}$ & $2.186 \mathrm{~d}$ & $13.548 \mathrm{~d}$ \\
Quicuio & $4.643 \mathrm{cde}$ & $3.394 \mathrm{~b}$ & $4.550 \mathrm{bc}$ & $3.399 \mathrm{bc}$ & $15.686 \mathrm{c}$ \\
Média & $5.038 \mathrm{~A}$ & $3.685 \mathrm{~B}$ & $5.043 \mathrm{~A}$ & $3.685 \mathrm{~B}$ & \\
\hline
\end{tabular}

Médias seguidas de letras minúsculas, diferentes na coluna, e maiúsculas, diferentes na linha, diferem entre si pelo teste de Tukey a 5\% de significância.

Como reflexo das maiores produções por corte, nota-se que a produção de fitomassa seca acumulada durante os quatro cortes seguiu a mesma tendência, sendo as cv. jiggs e Tifton 68, com 21.348 e $21.016 \mathrm{~kg} \mathrm{ha}^{-1}$, respectivamente, responsáveis pelas maiores produções $(\mathrm{P}<0,05)$, enquanto as cv. Hemártrhia roxinha e Quicuio se mostraram produtivamente inferiores $(13.548 \mathrm{e}$ $15.686 \mathrm{~kg} \mathrm{ha}^{-1}$, respectivamente) a todas as cultivares do gênero Cynodon.

A produtividade de cada forrageira é condicionada pelo seu material genético, pelo tipo de manejo empregado, pelas características climáticas e principalmente edáficas encontradas nelas (Nabinger et al., 2006). Tal fato explica a superioridade produtiva das cv. do gênero Cynodon em relação às demais, visto que são justamente conhecidas como cosmopolitas por serem extremamente tolerantes a variações de tipo e pH de solo (Hill et al., 2001).

Guimarães (2012), entre outros autores, destacam a superioridade produtiva da cv. Tifton 85 dada às características do seu ciclo, porém, em manejo de quatro cortes, não foi o que se observou. Dessa forma, a dinâmica do acúmulo de fitomassa aliado ao ciclo de cada cultivar se mostra fundamental para um planejamento forrageiro adequado.

De forma mais precisa, o ponto de corte ideal, que apresenta melhor relação entre produção e qualidade das forragens, é atingido com índice de 95\% de IL (Zanini et al., 2012). A Tab. 3 apresenta o IAF conforme o número de cortes.

Mais uma vez, a cv. Tifton 68 e a cv. Estrelaroxa mostraram superioridade significativa $(\mathrm{P}<0,05)$ em relação às demais cv. estudadas, enquanto a cv. Hemárthria roxinha obteve o menor IAF. Pereira et al. (2006) afirmam que a cv. Tifton 68 tem grande capacidade em interceptar ondas luminosas, dada a morfologia de suas folhas, as quais se tornam mais pronunciadas próximo ao ponto de pastejo, o que explica seu elevado índice de IAF. O oposto é observado com a cv. Hemárthria roxinha, a qual é caracterizada por possuir colmos e folhas muito finas (Carvalho et al., 2010). Já a cv. Estrelaroxa se mostra eficiente na interceptação luminosa e capaz de apresentar alto IAF devido a seu estande relativamente aberto.

Tabela 3. Índice de área foliar (IAF) das cultivares perenes, de acordo com cada corte em que o material fora submetido

\begin{tabular}{lccccc}
\hline Forrageira & \multicolumn{5}{c}{ Índice de área foliar, índice } \\
\cline { 2 - 5 } & $1^{\circ}$ corte & $2^{\circ}$ corte & $3^{\circ}$ corte & 4 corte & Média \\
\hline Estrela-roxa & $6,3 \mathrm{ab}$ & $5,6 \mathrm{ab}$ & $5,8 \mathrm{~b}$ & $4,6 \mathrm{~cd}$ & 5,6 \\
Coast-cross & $5,3 \mathrm{~d}$ & $5,4 \mathrm{~b}$ & $5,8 \mathrm{~b}$ & $4,2 \mathrm{~d}$ & 5,2 \\
Tifton 68 & $6,4 \mathrm{a}$ & $5,9 \mathrm{a}$ & $6,8 \mathrm{a}$ & $6,5 \mathrm{a}$ & 6,4 \\
Tifton 85 & $5,8 \mathrm{bc}$ & $5,6 \mathrm{ab}$ & $5,7 \mathrm{~b}$ & $5,3 \mathrm{~b}$ & 5,6 \\
Jiggs & $5,3 \mathrm{~d}$ & $5,6 \mathrm{ab}$ & $5,9 \mathrm{~b}$ & $5,9 \mathrm{a}$ & 5,7 \\
Hemártrhia roxinha & $5,6 \mathrm{~cd}$ & $4,6 \mathrm{c}$ & $5,6 \mathrm{~b}$ & $4,2 \mathrm{~d}$ & 5,0 \\
Quicuio & $5,2 \mathrm{~d}$ & $5,1 \mathrm{~b}$ & $6,6 \mathrm{a}$ & $4,9 \mathrm{bc}$ & 5,5 \\
Média & $5,7 \mathrm{~B}$ & $5,4 \mathrm{C}$ & $6,0 \mathrm{~A}$ & $5,1 \mathrm{D}$ & \\
\hline
\end{tabular}

Médias seguidas de letras minúsculas, diferentes na coluna, e maiúsculas, diferentes na linha, diferem entre si pelo teste de Tukey a 5\% de significância. 
Ainda na Tab. 3, nota-se que a cv. Tifton 68 apresentou-se invariável em relação ao IAF assim como para a produtividade de fitomassa seca por cortes (Tab. 2), demonstrando ser uma cultivar com alta habilidade em manter constância produtiva.

As variações de IAF entre os cortes realizados são fruto da dinâmica de crescimento e de processos fisiológicos naturais da planta (Silva et al., 2015), sendo, nesse caso, o terceiro corte responsável pela maior média de IAF e o quarto pela menor (6,0 e 5,1, respectivamente). Da mesma forma, Fagundes et al. (2006) relataram diminuições nos índices de IAF com o avanço das estações mais frias do ano.

De uma maneira geral, a curva de rebrota é caracterizada por três fases, e, na primeira, o acúmulo de MS aumenta exponencialmente com o tempo. Essa fase é altamente influenciada pelas reservas de carboidratos solúveis da planta, pela disponibilidade de fatores de crescimento e pela área residual de folhas após o corte. A segunda fase apresenta acúmulos constantes de MS. Nessa fase, o dossel se aproxima da completa interceptação luminosa. Já na terceira fase, temse uma diminuição do acúmulo de MS, dado o início do aumento da senescência de folhas, e aumento do sombreamento das folhas inferiores (Hodgson et al., 1981).

Corroborando tal afirmativa, a média geral dos cortes da relação folha:colmo seguiu a mesma tendência, em que o terceiro corte gerou a maior proporção de folhas tanto no estrato superior quanto no inferior $(\mathrm{P}<0,05)$.

De forma complementar, a Tab. 4 apresenta a relação folha:colmo do estrato superior, esta intimamente ligada à qualidade da forragem e do estrato inferior, fundamental para a rebrota.

Tabela 4. Relação folha:colmo dos estratos superior e inferior das cultivares perenes, conforme sistema de cortes

\begin{tabular}{|c|c|c|c|c|c|}
\hline \multirow[t]{2}{*}{ Forrageira } & \multicolumn{4}{|c|}{ Sistema de avaliação } & \multirow[t]{2}{*}{ Média } \\
\hline & 1 corte & $2^{\circ}$ corte & $3^{\circ}$ corte & $4^{\circ}$ corte & \\
\hline & \multicolumn{5}{|c|}{ Estrato superior } \\
\hline Estrela-roxa & $1,69 \mathrm{bc}$ & $1,71 \mathrm{~cd}$ & $1,93 \mathrm{bc}$ & $1,68 \mathrm{~b}$ & 1,74 \\
\hline Coast-cross & $2,05 \mathrm{ab}$ & $1,99 \mathrm{bc}$ & $2,26 \mathrm{~b}$ & $1,59 \mathrm{~b}$ & 1,97 \\
\hline Tifton 68 & $2,01 \mathrm{ab}$ & $2,29 \mathrm{ab}$ & $1,51 \mathrm{~cd}$ & $1,42 \mathrm{bc}$ & 1,81 \\
\hline Tifton 85 & $2,23 \mathrm{a}$ & $2,44 \mathrm{a}$ & $4,51 \mathrm{a}$ & $4,03 \mathrm{a}$ & 3,03 \\
\hline Jiggs & $1,13 \mathrm{~d}$ & $1,29 \mathrm{~d}$ & $1,28 \mathrm{~d}$ & $1,01 \mathrm{c}$ & 1,18 \\
\hline Hemártrhia roxinha & $1,38 \mathrm{~cd}$ & $2,29 \mathrm{ab}$ & $1,42 \mathrm{~d}$ & $1,22 \mathrm{bc}$ & 1,58 \\
\hline Quicuio & $1,30 \mathrm{~cd}$ & 0,99 e & $1,09 \mathrm{~d}$ & $1,08 \mathrm{c}$ & 1,12 \\
\hline \multirow[t]{2}{*}{ Média } & $1,68 \mathrm{~B}$ & $1,86 \mathrm{AB}$ & $2,00 \mathrm{~A}$ & $1,72 \mathrm{~B}$ & \\
\hline & \multicolumn{5}{|c|}{ Estrato inferior } \\
\hline Estrela-roxa & $0,33 \mathrm{~cd}$ & $0,33 \mathrm{~d}$ & $0,36 \mathrm{c}$ & $0,23 \mathrm{c}$ & 0,31 \\
\hline Coast-cross & $0,49 \mathrm{bc}$ & $0,51 \mathrm{cbd}$ & $0,95 \mathrm{~b}$ & $0,42 \mathrm{bc}$ & 0,59 \\
\hline Tifton 68 & $0,53 \mathrm{bc}$ & $0,53 \mathrm{bcd}$ & $0,36 \mathrm{c}$ & $0,42 \mathrm{bc}$ & 0,46 \\
\hline Tifton 85 & $0,59 \mathrm{~b}$ & $0,60 \mathrm{bc}$ & $0,55 \mathrm{c}$ & $0,52 \mathrm{~b}$ & 0,57 \\
\hline Jiggs & $0,25 \mathrm{~d}$ & $0,37 \mathrm{~cd}$ & $0,49 \mathrm{c}$ & $0,44 \mathrm{bc}$ & 0,39 \\
\hline Hemártrhia roxinha & $0,37 \mathrm{bcd}$ & $0,62 \mathrm{~b}$ & $1,19 \mathrm{a}$ & $1,29 \mathrm{a}$ & 0,87 \\
\hline Quicuio & $1,13 \mathrm{a}$ & $1,02 \mathrm{a}$ & $1,03 \mathrm{ab}$ & $1,00 \mathrm{a}$ & 1,05 \\
\hline Média & $0,53 \mathrm{~B}$ & $0,57 \mathrm{~B}$ & $0,70 \mathrm{~A}$ & $0,62 \mathrm{AB}$ & \\
\hline
\end{tabular}

Médias seguidas de letras minúsculas, diferentes na coluna, e maiúsculas, diferentes na linha, diferem entre si pelo teste Tukey a 5\% de significância.

Houve interação significativa $(\mathrm{P}<0,05)$ entre as espécies forrageiras e os números de cortes, tanto para o estrato superior quanto para o inferior, sendo a cv. Tifton 85 responsável por apresentar a maior relação folha:colmo no estrato superior em todos os cortes. Já a cv. Quicuio apresentou maior proporção de folhas em relação a colmos no estrato inferior em todos os cortes, fato esse explicado pela própria característica de altura dela. Corroborando tal hipótese, Padilha (2013) encontrou para essa cultivar altura média de dossel de $25 \mathrm{~cm}$ apenas.

Ademais, as cultivares do gênero Cynodon aumentam a relação folha:colmo quando atingem altura superior a $14 \mathrm{~cm}$ (Marchesan et al., 2013) por possuírem característica estolonífera de 
crescimento prostrado, tendo o colmo, em sua grande maioria, no estrato inferior.

De fato, a relação folha:colmo no estrato superior tende a ser maior quando comparado ao estrato inferior, haja vista que, com o avanço do ciclo, os colmos se alongam em busca de maior luminosidade, tendência essa observada no presente estudo.

Quanto à baixa relação de folhas no estrato inferior, cabe destacar que essa é uma característica do regime de cortes aplicado ou de um sistema de pastejo rotativo, visto que, nesses casos, há um período de descanso maior, o que permite que haja competição entre as plantas por luz de forma mais facilitada, e, assim, uma diminuição do número de perfilhos, com crescimento vertical deles (Silva et al., 2015).

Tal fato também interfere diretamente na relação entre estratos superior e inferior, conforme observado na Tab. 5. Na média geral dos dados, nota-se que a cv. Quicuio foi a única que apresentou relação menor que um $(0,95$ de estrato superior para 1,00 de estrato inferior), demonstrando uma proporção menor de estrato superior em relação ao inferior.

$\mathrm{O}$ estrato superior da pastagem compreende a maior concentração de carboidratos solúveis da planta, ou seja, quanto maior for a proporção desse estrato, melhor será a qualidade nutricional da forragem. Dessa forma, dados das Tab. 4 e 5 permitem sugerir que o manejo de cortes simulando um sistema de pastejo rotativo oferece condições para que os animais consumam continuamente um alimento de qualidade superior, quando comparado ao pastejo intermitente.

A qualidade nutricional da pastagem, por vezes, é mais importante do que a própria capacidade produtiva dela, pois pastagens altamente produtivas e de baixa qualidade nutricional não atendem às necessidades energéticas e proteicas dos animais (Marchesan et al., 2013).

Tabela 5. Relação estratos superior e inferior, na matéria verde, das cultivares perenes, conforme número de cortes

\begin{tabular}{lccccc}
\hline Forrageira & \multicolumn{5}{c}{ Relação estrato superior e estrato inferior } \\
\cline { 2 - 6 } & 1 corte & 2 corte & 3 corte & 4 corte & Média \\
\hline Estrela-roxa & $2,37 \mathrm{a}$ & $2,66 \mathrm{a}$ & $2,50 \mathrm{a}$ & $2,31 \mathrm{a}$ & 2,45 \\
Coast-cross & $2,73 \mathrm{a}$ & $2,30 \mathrm{ab}$ & $2,04 \mathrm{ab}$ & $2,06 \mathrm{a}$ & 2,26 \\
Tifton 68 & $2,26 \mathrm{ab}$ & $1,80 \mathrm{bc}$ & $2,35 \mathrm{a}$ & $2,19 \mathrm{a}$ & 2,14 \\
Tifton 85 & $1,40 \mathrm{c}$ & $1,40 \mathrm{c}$ & $1,17 \mathrm{~d}$ & $1,20 \mathrm{~b}$ & 1,29 \\
Jiggs & $1,65 \mathrm{bc}$ & $2,03 \mathrm{~b}$ & $1,91 \mathrm{ab}$ & $2,04 \mathrm{a}$ & 1,90 \\
Hemártrhia roxinha & $1,91 \mathrm{abc}$ & $1,38 \mathrm{c}$ & $1,31 \mathrm{c}$ & $0,47 \mathrm{~d}$ & 1,12 \\
Quicuio & $0,78 \mathrm{~d}$ & $0,93 \mathrm{~d}$ & $1,49 \mathrm{~b}$ & $0,75 \mathrm{c}$ & 0,95 \\
Média & $1,72 \mathrm{~A}$ & $1,67 \mathrm{~A}$ & $1,74 \mathrm{~A}$ & $1,34 \mathrm{~B}$ & \\
\hline
\end{tabular}

Médias seguidas de letras minúsculas, diferentes na coluna, e maiúsculas, diferentes na linha, diferem entre si pelo teste Tukey a 5\% de significância.

Ademais, a qualidade nutricional de uma pastagem sofre influência direta do momento no qual é colhida ou consumida, como verificado nas Tab. 6 e 7. As mesmas tabelas ainda apresentam os níveis de PB, FDN e FDA das cultivares perenes em seus estratos superior e inferior, respectivamente.

Pontualmente, a média geral dos dados da Tab. 6 demonstra que a cv. Tifton 85 apresentou o maior teor de PB entre as cultivares estudadas, sendo superior estatisticamente $(\mathrm{P}<0,05)$ no primeiro, segundo e quarto cortes, enquanto no terceiro corte a cv. Coast-cross apresentou o maior teor proteico.

O maior nível de PB da cv. Tifton 85 condiz com os achados da Tab. 4, em que ela apresentou a maior relação folha:colmo no estrato superior, sendo justamente as folhas, responsáveis pelo maior acúmulo de PB das plantas (Taiz e Zeiger, 1991). 
Tabela 6. Teores médios de proteína bruta, fibra em detergente neutro e fibra em detergente ácido do estrato superior das cultivares perenes, conforme sistema de cortes

\begin{tabular}{|c|c|c|c|c|c|}
\hline \multirow[t]{2}{*}{ Forrageira } & \multicolumn{4}{|c|}{ Sistema de avaliação } & \multirow[t]{2}{*}{ Média } \\
\hline & $1^{\circ}$ corte & $2^{\circ}$ corte & $3^{\circ}$ corte & $4^{\circ}$ corte & \\
\hline & \multicolumn{5}{|c|}{ Proteína bruta, $\%$ na MS } \\
\hline Estrela-roxa & $13,08 \mathrm{c}$ & $14,24 \mathrm{~b}$ & $13,57 \mathrm{bc}$ & $12,21 \mathrm{c}$ & 14,02 \\
\hline Coast-cross & $16,11 \mathrm{~b}$ & $15,79 \mathrm{~b}$ & $15,67 \mathrm{a}$ & $14,51 \mathrm{~b}$ & 15,52 \\
\hline Tifton 68 & $16,68 \mathrm{~b}$ & $15,48 \mathrm{~b}$ & $15,13 \mathrm{abc}$ & $12,42 \mathrm{c}$ & 14,17 \\
\hline Tifton 85 & 17,99 a & $18,29 \mathrm{a}$ & $13,68 \mathrm{bc}$ & $18,56 \mathrm{a}$ & 17,13 \\
\hline Jiggs & $14,21 \mathrm{c}$ & $14,13 \mathrm{~b}$ & $13,76 \mathrm{bc}$ & $13,81 \mathrm{bc}$ & 14,73 \\
\hline Hemártrhia roxinha & $8,22 \mathrm{~d}$ & $9,59 \mathrm{c}$ & $8,54 \mathrm{~d}$ & $11,96 \mathrm{~d}$ & 9,44 \\
\hline Quicuio & $8,79 \mathrm{~d}$ & $14,87 \mathrm{~b}$ & $15,52 \mathrm{ab}$ & $15,04 \mathrm{~b}$ & 13,56 \\
\hline \multirow[t]{2}{*}{ Média } & $13,58 \mathrm{~B}$ & $15,05 \mathrm{~A}$ & $13,70 \mathrm{~B}$ & $13,99 \mathrm{~B}$ & \\
\hline & \multicolumn{5}{|c|}{ Fibra em detergente neutro, $\%$ na MS } \\
\hline Estrela-roxa & $70,45 \mathrm{~b}$ & $62,64 \mathrm{~d}$ & $71,97 \mathrm{a}$ & $70,87 \mathrm{a}$ & 68,98 \\
\hline Coast-cross & $73,35 \mathrm{ab}$ & $68,73 \mathrm{abc}$ & $70,35 \mathrm{a}$ & 71,99 a & 71,10 \\
\hline Tifton 68 & $72,64 \mathrm{ab}$ & $65,57 \mathrm{~cd}$ & $71,15 \mathrm{a}$ & $71,35 \mathrm{a}$ & 70,18 \\
\hline Tifton 85 & $72,27 \mathrm{ab}$ & $67,37 \mathrm{abc}$ & $68,75 \mathrm{a}$ & $69,27 \mathrm{a}$ & 69,42 \\
\hline Jiggs & 75,16 a & $70,34 \mathrm{ab}$ & $71,80 \mathrm{a}$ & $71,09 \mathrm{a}$ & 71,10 \\
\hline Hemártrhia roxinha & $75,14 \mathrm{ab}$ & $66,29 \mathrm{bcd}$ & $72,52 \mathrm{a}$ & $68,59 \mathrm{a}$ & 69,39 \\
\hline Quicuio & $71,21 \mathrm{ab}$ & $71,42 \mathrm{a}$ & $70,02 \mathrm{a}$ & 68,99 a & 70,41 \\
\hline \multirow[t]{2}{*}{ Média } & $72,89 \mathrm{~A}$ & $67,48 \mathrm{C}$ & $70,94 \mathrm{AB}$ & $70,31 \mathrm{~B}$ & \\
\hline & \multicolumn{5}{|c|}{ Fibra em detergente ácido, $\%$ na MS } \\
\hline Estrela-roxa & $33,43 \mathrm{bc}$ & $31,08 \mathrm{~b}$ & $30,67 \mathrm{a}$ & $30,13 \mathrm{c}$ & 31,33 \\
\hline Coast-cross & $34,70 \mathrm{a}$ & $34,85 \mathrm{a}$ & $33,06 \mathrm{a}$ & $31,45 \mathrm{bc}$ & 33,51 \\
\hline Tifton 68 & $30,52 \mathrm{c}$ & $32,02 \mathrm{~b}$ & $32,08 \mathrm{a}$ & $33,80 \mathrm{~b}$ & 32,10 \\
\hline Tifton 85 & $31,88 \mathrm{bc}$ & $31,05 \mathrm{~b}$ & $30,68 \mathrm{a}$ & $36,70 \mathrm{a}$ & 32,58 \\
\hline Jiggs & $32,46 \mathrm{bc}$ & $31,97 \mathrm{~b}$ & $32,17 \mathrm{a}$ & $32,46 \mathrm{bc}$ & 32,26 \\
\hline Hemártrhia roxinha & $34,68 \mathrm{~b}$ & $36,21 \mathrm{a}$ & $33,20 \mathrm{a}$ & $33,28 \mathrm{~b}$ & 34,34 \\
\hline Quicuio & $33,93 \mathrm{~b}$ & $27,41 \mathrm{c}$ & $31,88 \mathrm{a}$ & $31,96 \mathrm{bc}$ & 31,29 \\
\hline Média & $33,08 \mathrm{~A}$ & $32,08 \mathrm{~A}$ & $31,96 \mathrm{~A}$ & $32,79 \mathrm{~A}$ & \\
\hline
\end{tabular}

Médias seguidas de letras minúsculas, diferentes na coluna, e maiúsculas, diferentes na linha, diferem entre si pelo teste Tukey a 5\% de significância.

O menor teor de $\mathrm{PB}$ foi observado na cv. Hemártrhia roxinha $(9,44 \%)$, sendo esse um valor considerado abaixo do ótimo para forragens de verão (10,7\%) (Hanisch e Fonseca, 2011). Porém, esse valor está acima do encontrado por Postiglioni et al. (2002) em condições de campo ( $4 \%$ a $6 \%$ ). Nota-se ainda que, na média geral dos cortes, o maior nível de PB observado foi no segundo corte $(15,05 \%)$.

Ainda na Tab. 6, observa-se que os teores de FDN do estrato superior não apresentaram grandes variações entre os cortes, como nas cv. Coast-cross, Tifton 85, Jiggs e Quicuio, que não diferiram estatisticamente $(\mathrm{P}>0,05)$ em todos os cortes. Comportamento semelhante foi observado no estrato inferior para as cv. Jiggs, Hemártrhia roxinha e Quicuio (Tab. 7).
Na média geral dos dados do estrato superior, o primeiro e o terceiro corte apresentaram menor $(\mathrm{P}<0,05)$ teor de FDN, enquanto o segundo corte foi o que gerou menor FDN no estrato inferior.

Ainda na Tab. 7, observa-se que as cv. Coastcross e Tifton 85 apresentaram os maiores $(\mathrm{P}<0,05)$ teores de $\mathrm{PB}$ em relação às demais no primeiro corte. Nesse mesmo corte, foi observado o menor teor de PB das médias de cortes $(8,96 \%)$, que diferiram estatisticamente do segundo, terceiro e quarto cortes $(10,08 ; 10,45$ e 10,04 ; respectivamente).

O baixo teor médio de PB encontrado no estrato inferior retrata a baixa concentração de folhas nessa porção, como destacado por Nabinger (2006). Porém, Neres et al. (2012) enfatizam que bovinos necessitam de um mínimo de 7\% de PB em sua dieta para que haja condições favoráveis 
para mantença e para o perfeito desempenho ruminal; dessa forma, todas as cultivares avaliadas, em todos os cortes, suprem essa exigência.

Em relação à fração FDN das forragens, a cv. Estrela-roxa diferiu significativamente $(\mathrm{P}<0,05)$ das demais cultivares no primeiro corte, com
$71,01 \%$ de FDN na base seca. Já no terceiro e no quarto corte, não foi observada diferença significativa $(\mathrm{P}>0,05)$ entre as forragens. Sugerese que o intervalo entre cortes possa ter influenciado os teores de FDN, visto que, com o avanço do ciclo, ocorre maior deposição de carboidratos estruturais na parede celular da planta (Oliveira et al., 2013).

Tabela 7. Teores médios de proteína bruta, fibra em detergente neutro e fibra em detergente ácido do estrato inferior das cultivares perenes, conforme sistema de cortes

\begin{tabular}{|c|c|c|c|c|c|}
\hline \multirow[t]{2}{*}{ Forrageira } & \multicolumn{4}{|c|}{ Sistema de avaliação } & \multirow[t]{2}{*}{ Média } \\
\hline & $1^{\circ}$ corte & $2^{\circ}$ corte & 3 corte & $4^{\circ}$ corte & \\
\hline & \multicolumn{5}{|c|}{ Proteína bruta, $\%$ na MS } \\
\hline Estrela-roxa & $7,37 \mathrm{~b}$ & $10,57 \mathrm{~b}$ & $9,91 \mathrm{bc}$ & 9,09 bc & 9,23 \\
\hline Coast-cross & $10,97 \mathrm{a}$ & $12,08 \mathrm{a}$ & $11,80 \mathrm{ab}$ & $11,56 \mathrm{a}$ & 11,60 \\
\hline Tifton 68 & $8,74 \mathrm{~b}$ & $8,79 \mathrm{c}$ & $8,40 \mathrm{c}$ & 8,98 bc & 8,37 \\
\hline Tifton 85 & $11,87 \mathrm{a}$ & $12,11 \mathrm{a}$ & $11,14 \mathrm{ab}$ & $12,95 \mathrm{a}$ & 12,02 \\
\hline Jiggs & $9,02 \mathrm{~b}$ & $10,43 \mathrm{~b}$ & $10,42 \mathrm{~b}$ & $9,73 \mathrm{bc}$ & 9,90 \\
\hline Hemártrhia roxinha & $7,36 \mathrm{~b}$ & $7,71 \mathrm{~d}$ & $8,91 \mathrm{bc}$ & $8,17 \mathrm{c}$ & 8,04 \\
\hline Quicuio & $7,40 \mathrm{~b}$ & $8,90 \mathrm{bc}$ & $12,59 \mathrm{a}$ & $9,78 \mathrm{bc}$ & 9,67 \\
\hline \multirow[t]{2}{*}{ Média } & $8,96 \mathrm{~B}$ & $10,08 \mathrm{~A}$ & $10,45 \mathrm{~A}$ & $10,04 \mathrm{~A}$ & \\
\hline & \multicolumn{5}{|c|}{ Fibra em detergente neutro, $\%$ na MS } \\
\hline Estrela-roxa & $71,01 \mathrm{~b}$ & $69,09 \mathrm{~cd}$ & $74,87 \mathrm{a}$ & $73,63 \mathrm{a}$ & 70,89 \\
\hline Coast-cross & $76,16 \mathrm{a}$ & $71,84 \mathrm{bc}$ & $72,87 \mathrm{a}$ & 74,99 a & 73,97 \\
\hline Tifton 68 & $75,13 \mathrm{a}$ & $68,18 \mathrm{~d}$ & $75,11 \mathrm{a}$ & $72,72 \mathrm{a}$ & 72,79 \\
\hline Tifton 85 & $77,32 \mathrm{a}$ & $70,90 \mathrm{bcd}$ & $74,52 \mathrm{a}$ & $74,59 \mathrm{a}$ & 74,33 \\
\hline Jiggs & $76,23 \mathrm{a}$ & $73,33 \mathrm{ab}$ & $72,67 \mathrm{a}$ & $72,65 \mathrm{a}$ & 73,72 \\
\hline Hemártrhia roxinha & $74,63 \mathrm{a}$ & $74,98 \mathrm{a}$ & $73,46 \mathrm{a}$ & 74,03 a & 74,28 \\
\hline Quicuio & $74,62 \mathrm{a}$ & $75,40 \mathrm{a}$ & $75,68 \mathrm{a}$ & $73,72 \mathrm{a}$ & 74,85 \\
\hline \multirow[t]{2}{*}{ Média } & $75,02 \mathrm{~A}$ & $71,96 \mathrm{~B}$ & $74,17 \mathrm{~A}$ & $73,76 \mathrm{~A}$ & \\
\hline & \multicolumn{5}{|c|}{ Fibra em detergente ácido, \% na MS } \\
\hline Estrela-roxa & $36,03 \mathrm{~b}$ & $39,19 \mathrm{a}$ & $36,84 \mathrm{~b}$ & $34,65 \mathrm{ab}$ & 36,68 \\
\hline Coast-cross & $35,06 \mathrm{~b}$ & $37,40 \mathrm{a}$ & $37,63 \mathrm{ab}$ & $38,43 \mathrm{a}$ & 37,13 \\
\hline Tifton 68 & $34,77 \mathrm{~b}$ & $35,40 \mathrm{a}$ & $35,43 \mathrm{~b}$ & $39,20 \mathrm{a}$ & 36,20 \\
\hline Tifton 85 & $37,43 \mathrm{~b}$ & $36,14 a$ & $40,53 \mathrm{ab}$ & $38,89 \mathrm{a}$ & 38,25 \\
\hline Jiggs & $35,64 \mathrm{~b}$ & $35,45 \mathrm{a}$ & $35,41 \mathrm{~b}$ & $35,37 \mathrm{ab}$ & 35,47 \\
\hline Hemártrhia roxinha & $46,42 \mathrm{a}$ & $36,09 \mathrm{a}$ & $42,64 \mathrm{a}$ & 39,72 a & 41,21 \\
\hline Quicuio & $34,06 \mathrm{~b}$ & $33,08 \mathrm{~b}$ & $38,24 \mathrm{ab}$ & $32,04 \mathrm{~b}$ & 34,35 \\
\hline Média & $37,06 \mathrm{~A}$ & $35,74 \mathrm{~A}$ & $38,03 \mathrm{~A}$ & $36,58 \mathrm{~A}$ & \\
\hline
\end{tabular}

Médias seguidas de letras minúsculas, diferentes na coluna, e maiúsculas, diferentes na linha, diferem entre si pelo teste Tukey a $5 \%$ de significância.

Corroborando tal afirmação, Andrade et al. (2007) relatam que as maiores mudanças na composição das plantas forrageiras são aquelas decorrentes da sua maturidade. A maioria das espécies tendem a um declínio do seu valor nutritivo com o avanço do ciclo, resultado esse da menor relação folha:colmo e da crescente lignificação da parede celular.

Soares et al. (2009), ao avaliarem o comportamento da Hemárthria, encontraram valores de FDN próximos aos do presente estudo, entre $71 \%$ e $73 \%$, em avaliações efetuadas em tempo fixo de corte. Os mesmos autores afirmam que esses valores são considerados aceitáveis em plantas forrageiras, os quais ainda enfatizam a relação direta da FDN com o consumo pelo animal, e da FDA com a digestibilidade da forragem.

Numericamente, os teores de FDA do estrato inferior se mostraram maiores aos do estrato 
superior, pois a fração colmo (maior no estrato inferior em relação ao superior, como apresentado na Tab. 4) possui maiores concentrações de celulose e lignina, dada sua característica de sustentação (Taiz e Zeiger, 1991).

As diferenças entre FDN e FDA nos estratos superior e inferior são descritas comumente em plantas de crescimento ereto e cespitoso, como observado por Silva et al. (2007) e Rêgo et al. (2002). Todavia, os valores encontrados no presente trabalho mostram que plantas de crescimento prostrado apresentaram a mesma tendência.

\section{CONCLUSÃO}

As cv. Jiggs e Tifton 68 apresentaram a maior produção de fitomassa acumulada. Já a cv. Tifton 85 foi responsável pelos maiores níveis de $\mathrm{PB}$, enquanto os teores de carboidratos estruturais mantiveram padrão constante, tanto no estrato superior quanto no inferior, em sistema de quatro cortes sucessivos.

\section{AGRADECIMENTOS}

Os autores agradecem à Capes, pela bolsa de estudo concedida entre os anos de 2012 e 2014, o que tornou possível a realização desta obra.

\section{REFERÊNCIAS}

ANDRADE R.L.R.; RODRIGUES, T.J.D.; REIS, R.A.; SOARES FILHO, C.V. Produção de massa seca e composição química de cinco cultivares de Cynodon. Acta Sci., Anim. Sci., v.28, p.251-258, 2007.

BIRCHAM, J.S. Herbage growth and utilization under continuous stocking management. 1981. 384f. Thesis (Ph.D) - University of Edinburgh, Edinburgh.

BURTON, G.W.; MONSON, W.G. Registration of 'Tifton 68' bermudagrass. Crop Sci., v.24, p.1211-1219, 1993.

CARVALHO, P.C.F. Característica produtiva e estrutural de pastos mistos de aveia e azevém manejados em quatro alturas sob lotação contínua. Rev. Bras. Zootec., v.39, p.1857-1865, 2010 .

Arq. Bras. Med. Vet. Zootec., v.68, n.3, p.785-794, 2016
FAGUNDES, J.L.; FONSECA, D.M.; MISTURA, C. et al. Características morfogênicas e estruturais do capim braquiária em pastagem adubada com nitrogênio avaliadas nas quatro estações do ano. Rev. Bras. Zootec., v.35, p.21-29, 2006.

GOERING, H.K.; VAN SOEST, P.J. Forage fiber analysis: apparatus reagents, procedures and some applications. [s.l.]: United States Department Agriculture / Agricultural Research Services, 1970. (Agricultural Handbook, n.379).

GUIMARÃES, M.S. Desempenho produtivo, análise de crescimento e características estruturais do dossel de dois capins do gênero Cynodon sob duas estratégias de pastejo intermitente. 2012. 81f. Tese (Doutorado) Escola Superior de Agricultura "Luiz de Queiroz", Universidade de São Paulo, Piracicaba, SP.

HANISCH, A.L.; FONSECA, J.A. Características produtivas e qualitativas de sete forrageiras perenes de verão sob adubação orgânica e mineral. Rev. Verde, v.6, n.4, p.1-6, 2011

HILL, G.M.; GATES, R.N.; WEST J.W. Advances in bermudagrass research involving new cultivars for beef and dairy production. $J$. Anim. Sci., v.79, p E48-E58, 2001.

HODGSON, J. Variations in the surface characteristics of the sward and short-term rate of herbage intake by calves and lambs. Grass Forage Sci., v.36, p.49-57, 1981.

MARCHESAN, R.; PARIS, W.; ZIECH, M.F. et al. Produção e composição químicabromatológica de Tifton 85 (Cynodon dactylon L. Pers) sob pastejo contínuo no período hibernal. Semina: Ciênc. Agr., v.34, p.19351944, 2013.

NABINGER, C.; DALL'AGNOL, M.; CARVALHO, P.C.F. Biodiversidade e produtividade em pastagens. In: SIMPÓSIO SOBRE MANEJO DA PASTAGEM, 23. 2006, Piracicaba. Anais... Piracicaba: Fundação de Estudos Agrários Luiz de Queiroz, 2006. p.87138.

NERES, M.A.; CASTAGNARA, D.D.; SILVA, F.B. et al. Características produtivas, estruturais e bromatológicas dos capins Tifton 85 e Piatã e do feijão-guandu cv. Super $\mathrm{N}$, em cultivo singular ou em associação. Ciênc. Rural, v.42, p.862-869, 2012. 
OFFICIAL methods of analysis. 16.ed. Washington, D.C.: AOAC, 1995. 2000p.

OLIVEIRA, E.R.; MONÇÃO, F.P.; GOES, R.H.T.B. et al. Degradação ruminal da fibra em detergente neutro de gramíneas do gênero Cynodon spp em quatro idades de corte. Rev. Agrarian, v.6, p.205-214, 2013.

PADILHA, D.A. Acúmulo de forragem $e$ composição química em pastos de capim-quicuiu submetidos a estratégias de lotação intermitente. 2013. 62f. Dissertação (Mestrado) - Centro de Ciências Agroveterinárias, Universidade do Estado de Santa Catarina, Lages.

PEDREIRA, C.G.S.; SILVA, S.C. Acúmulo de forragem durante a rebrotação de capim-xaraés submetido a três estratégias de desfolhação. Rev. Bras. Zootec., v.38, p.618-625, 2009.

PEREIRA, O.G.; OBEID, J.A.; JUNIOR, D.N., FONSECA, D.M. Simpósio sobre manejo estratégico da pastagem. In: SIMPÓSIO SOBRE MANEJO ESTRATÉGICO DA PASTAGEM, 3.. 2006, Viçosa, MG. Anais... Viçosa: UFV / DZO, 2006. 430p.

POSTIGLIONI, S.R.; MOLETTA, J.L.; BREN, L. Minerais proteinados por novilhos em pastagens de Hermarthria altíssima cv. Florida. In:REUNIÓN DEL GRUPO TÉCNICO EN FORRAJERAS DEL CONO SUR, 19., Mercedes, Corrientes, Argentina, 2002. Anais... Corrientes: [s.n.], 2002. 263p.

POTT, C.A.; MÜLLER, M.M.L.; BERTELLI, P.B. Adubação verde como alternativa agroecológica para recuperação da fertilidade do solo. Rev. Ambiência, v.3, p.51-63, 2007.

RÊGO, F.C.A.; CECATO, U.; CANTO, M.W. et al. Características morfológicas e índice de área foliar do capim-tanzânia (Panicum maximum Jacq. cv. Tanzânia-1) manejado em diferentes alturas, sob pastejo. Rev. Bras. Zootec., v.31, p.1931-1937, 2002.
SAS INSTITUTE. SAS Language reference. Version 6, Cary, NC: 1042 p. 1993.

SILVA, S.C., SBRISSIA, A.F., PEREIRA, L.E.T. Ecophysiology of C4 forage grassesunderstanding plant growth for optimising their use and management. Agriculture, v.5, p.598$625,2015$.

SILVA, S.C.D.; NASCIMENTO JÚNIOR, D.D. Avanços na pesquisa com plantas forrageiras tropicais em pastagens: características morfofisiológicas e manejo do pastejo. Rev. Bras. Zootec., v.36, p.122-138, 2007.

SOARES, A.B.; SARTOR L.R.; ADAMI, P.F. et al. Influência da luminosidade no comportamento de onze espécies forrageiras perenes de verão. Rev. Bras. Zootec., v.38, p.443-451, 2009.

TAIZ, L.; ZEIGER, E. Plant physiology. Califórnia: The Benjamin/Cummings Publishings Company, 1991. 565p.

VAN SOEST, P.J. Nutritional ecology of the ruminant. 2.ed. Ithaca: Comstock Publishing Associates, 1994. 415p.

VAN SOEST, P.J.; ROBERTTSON, J.B.; LEWIS, B.A. Methods for dietary fiber, neutral detergent fiber and non starch polysaccharides in relation to animal nutrition. J. Dairy Sci., v.74, p.3583-3597, 1991 .

ZANINI, G.D., SANTOS, G.T., SCHMITT, D. et al. Distribution of stem in the vertical structure of Aruana guineagrass and Annual ryegrass pastures subjected to rotational grazing by sheep. Ciênc. Rural, v.42, p.882-887, 2012. 